\title{
Superradiance Transition in Photosynthetic Light-Harvesting Complexes
}

\author{
Giuseppe L. Celardo, ${ }^{*}{ }^{\dagger, \perp}$ Fausto Borgonovi, ${ }^{\dagger, \perp}$ Marco Merkli, ${ }^{\ddagger}$ Vladimir I. Tsifrinovich, ${ }^{\S}$ \\ and Gennady P. Berman"
}
${ }^{\dagger}$ Dipartimento di Matematica e Fisica and Interdisciplinary Laboratories for Advanced Materials Physics, Università Cattolica, via Musei 41, 25121 Brescia, Italy
${ }^{\perp}$ Istituto Nazionale di Fisica Nucleare, Sezione di Pavia, via Bassi 6, I-27100, Pavia, Italy
${ }^{\ddagger}$ Department of Mathematics and Statistics, Memorial University of Newfoundland, St. John’s, Newfoundland, Canada A1C 5S7
${ }^{\S}$ Department of Applied Physics, Polytechnic Institute of NYU, 6 MetroTech Center, Brooklyn, New York 11201, United States
${ }^{\|}$Theoretical Division, MS B213, Los Alamos National Laboratory, Los Alamos, New Mexico 87545, United States

ABSTRACT: We investigate the role of long-lasting quantum coherence in the efficiency of energy transport at room temperature in Fenna-Matthews-Olson photosynthetic complexes. The excitation energy transfer due to coupling of the lightharvesting complex to the reaction center ("sink") is analyzed using an effective nonHermitian Hamiltonian. We show that, as the coupling to the reaction center is varied, maximal efficiency in energy transport is achieved in the vicinity of the superradiance transition, characterized by a segregation of the imaginary parts of the eigenvalues of the effective non-Hermitian Hamiltonian. Our results demonstrate that the presence of the sink (which provides a quasi-continuum in the energy spectrum) is the dominant effect in the energy transfer which takes place even in the absence of a thermal bath. This approach allows one to study the effects of finite temperature and the effects of any coupling scheme to the reaction center. Moreover, taking into account a realistic electric dipole interaction, we show that the optimal distance from the reaction center to the

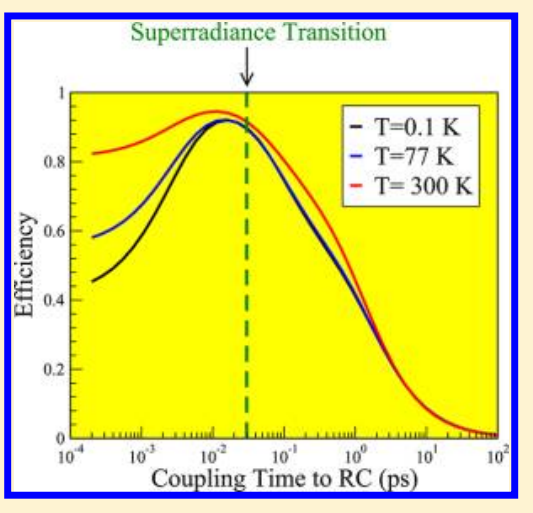
Fenna-Matthews-Olson system occurs at the superradiance transition, and we show that this is consistent with available experimental data.

\section{INTRODUCTION}

The annual amount of energy humans currently use is delivered to Earth by the Sun in a few hours! Since solar energy is very dilute, it is essential to transport the captured energy efficiently. Most natural photosynthetic systems consist of antenna complexes, which capture photons from the Sun and transport energy to a reaction center (RC). There, it is transformed into chemical energy via charge separation. Antenna complexes are able to transfer excitations to RCs with an efficiency exceeding $95 \%$. For a long time, it was thought that energy transfer in photosynthetic light-harvesting complexes occurs through classical processes, similar to random walks of the exciton to the RC. However, surprising evidence of coherent quantum energy transfer has been found recently. ${ }^{1,2}$ These findings raise two basic questions. How can coherence be maintained in complex biological systems at room temperature? Why is quantum coherence relevant to the efficiency of energy transfer?

The first question has been addressed in refs 3,4. We consider here the second one. It is known that quantum coherence can speed up energy transport through a quantum walk, which can be faster than a classical walk. ${ }^{5}$ Although the relevance of a mechanism similar to Dicke superradiance ${ }^{8}$ has been also pointed out, ${ }^{6,7}$ we focus here on a different feature of the "superradiance transition" (ST).${ }^{9,10}$ We show that ST is a dominant mechanism in an antenna complex described by discrete energy levels coupled to the $\mathrm{RC}$, modeled here by a sink having a continuum energy spectrum similar to what has been done in refs 3,5,11. On the other hand, the effects of the thermal bath lead only to small corrections to the energy transport in the vicinity of maximal efficiency. The antennasink coupling causes the appearance of a resonance width (inverse of lifetime) and an energy (Lamb) shift. For weak coupling strength, the resonance widths are roughly the same. However, if the coupling strength reaches a critical value, at which the resonance widths start to overlap, then a segregation of widths builds up. In this regime, almost the entire (summed up) decay width is allocated to just a few short-lived "superradiant states", while all other states are long-lived (and effectively decoupled from the environment). We call this segregation the "Superradiance Transition". This effect has been studied using random matrix theory, ${ }^{12,13}$ in nuclear physics, ${ }^{14}$ for microwave billiards, ${ }^{15}$ and in paradigmatic models of coherent quantum transport. ${ }^{16,17}$ It was shown in ref 16 that,

Received: March 19, 2012

Revised: September 5, 2012

Published: October 1, 2012 
in a realistic model for quantum transport, maximum transmission is achieved at ST.

In this paper, we focus on transport properties of the FennaMatthews-Olson (FMO) complex, found in green sulfur bacteria. This complex, one of the most studied in the literature, ${ }^{3-5,18}$ acts as a conductor for energy transport between the antenna system and the RC. The FMO complex is a dissipative open quantum system which interacts with the thermal bath provided by the protein environment. Here, we take an effective non-Hermitian Hamiltonian approach ${ }^{9,10,19}$ and study the ST as a function of the coupling to the RC and the thermal bath, due to phonons. The phonon bath induces dephasing and dissipation, and we take both effects into account using two different models for the phonon bath. Since $\mathrm{ST}$ is due to quantum coherence, we address here two main issues: (i) whether its effects can survive in the presence of dephasing induced by the phonon bath at room temperature, and (ii) how ST depends on the strength of the coupling between the FMO and the RC. It has been shown recently that maximal transport efficiency for the FMO complex is achieved near a critical coupling to the RC. ${ }^{20}$ However, so far, the dependence of this critical coupling on the parameters of the FMO and the RC has not been determined. We compute this critical coupling analytically and show that it corresponds to the ST. We demonstrate that the quantum coherent effect of ST, even taking into account dephasing and relaxation, determines the maximal transport efficiency at room temperature. Indeed, the ST is due to coherent constructive interference between the various paths to the RC, thus enhancing the rate of energy transfer. Finally, with the aid of the non-Hermitian Hamiltonian approach, we consider a realistic coupling between the FMO complex and the RC, showing that the ST determines the optimal distance from the RC to the FMO system.

\section{MODEL FOR SUPERRADIANCE TRANSITION}

The FMO complex is a trimer, composed of identical subunits, each of which contains seven bacteriochlorophylls (BChl). ${ }^{21}$ Each subunit acts independently and can be modeled using a tight-binding Hamiltonian

$$
H_{0}=\sum_{i=1}^{7} E_{i}|i\rangle\langle i|+\sum_{i, j}\left(J_{i, j}|i\rangle\langle j|+h . c .\right)
$$

Here, $|i\rangle$ is the state in which the ith site is excited and the others are in the ground state. Since the solar energy is very dilute, we limit the description to a single excitation in the complex, as is commonly done in the literature. The numerical values of $E_{i}$ and $J_{i, j}$ have been taken from ref 3. Below, we take the matrix elements of $H_{0}$ expressed in $\mathrm{cm}^{-1}$

$$
\left(\begin{array}{lllllll}
200 & -87.7 & 5.5 & -5.9 & 6.7 & -13.7 & -9.9 \\
-87.7 & 320 & 30.8 & 8.2 & 0.7 & 11.8 & 4.3 \\
5.5 & 30.8 & 0 & -53.5 & -2.2 & -9.6 & 6 \\
-5.9 & 8.2 & -53.5 & 110 & -70.7 & -17 & -63.3 \\
6.7 & 0.7 & -2.2 & -70.7 & 270 & 81.1 & -1.3 \\
-13.7 & 11.8 & -9.6 & -17 & 81.1 & 420 & 39.7 \\
-9.9 & 4.3 & 6 & -63.3 & -1.3 & 39.7 & 230
\end{array}\right)
$$

The incident photon creates an electron-hole pair, called an exciton, which decays due to two processes: coupling to the electromagnetic field, i.e., emission of a photon (recombina- tion) with an associated decay time, $T_{1}$, and coupling to the RC with a decay time, $T_{1 r}$.

As is common in quantum optics, ${ }^{22}$ we describe this dissipative system with at most one excitation by states

$$
|\psi\rangle=\sum_{i=1}^{7} a_{i}|0\rangle \otimes|i\rangle+\sum_{c} \int \mathrm{d} E b_{c}(E)|c, E\rangle \otimes|g s\rangle
$$

where $|0\rangle$ is the vacuum state of the environment and $|c, E\rangle \otimes \mid$ $g s\rangle$ is the state with one excitation in the environment and none on the sites. Here, $c$ is the quantum number labeling channels (at energies $E$ ) in the environments. The reduced density matrix is obtained by tracing over the states $|0\rangle$ and $|c, E\rangle$

$$
\rho=\sum_{i, j} a_{i} a_{j}^{*}|i\rangle\left\langle j\left|+\left(1-\sum_{i}\left|a_{i}\right|^{2}\right)\right| g s\right\rangle\langle g s|
$$

which is an $8 \times 8$ matrix. However, $\langle g s|\rho| i\rangle=0$, since with the choice in eq 2 , we neglect the transitions $|i\rangle \rightarrow|g s\rangle$. Moreover, $\langle g s|\rho| g s\rangle$ is simply the loss of probability of excitation of the seven sites. Therefore, we restrict our considerations to the $7 \times$ 7 matrix $\langle i|\rho| j\rangle, 1 \leq i, j \leq 7$, which however does not have constant trace.

In order to compute the evolution of the reduced density matrix, we introduce an effective non-Hermitian Hamiltonian ${ }^{9,16,23}$ which in general can be written as $H_{\text {eff }}(E)=H_{0}+$ $\Delta(E)-i W(E)$, where $H_{0}$ is the Hermitian Hamiltonian of the system decoupled from the environments and $\Delta(E)$ and $W(E)$ are the induced energy shift and the dissipation, respectively. Neglecting the energy dependence and the energy shift, we have

$$
H_{\text {eff }}=H_{0}-i W \quad \text { with } \quad W_{i j}=\sum_{c} A_{i}^{c}\left(A_{j}^{c}\right)^{*}
$$

The real symmetric matrix, $W$, is given in terms of the bound-state continuum transition amplitudes, $A_{i}^{c}$, from the discrete state $i$ to the continuum channel $c$.

The Schrödinger equation and eq 2 result in the following equation for the coefficients, $a_{j}$ :

$$
i \hbar \dot{a}_{j}=\sum_{k=1}^{7}\left(H_{0 j k} a_{k}-i W_{j k} a_{k}\right)
$$

and from this, the master equation easily follows

$$
i \hbar \dot{\rho}_{j k}=\left[H_{0}, \rho\right]_{j k}-i \sum_{l=1}^{7}\left(W_{j} \rho_{l k}+\rho_{j l} W_{l k}\right)
$$

Under the standard assumption ${ }^{24,25}$ that each site is coupled to an independent (local) environment, with associated coupling time $T_{1}$, we have $A_{i}^{i}=\left(\hbar / 2 T_{1}\right)^{1 / 2}, i=1, . ., 7$. The site $i=3$ is the only one which is, in addition, coupled to the RC, giving rise to a decay time $T_{1 r}$. Then, $A_{3}^{8}=\left(\hbar / 2 T_{1 r}\right)^{1 / 2}$ (in this scheme, there are $7+1$ channels); for other channels, we have $A_{i}^{\mathrm{c}}=0$. In eq 6 , we take into account the following: (i) the interaction between the FMO and the RC through the time $T_{1 r}$ and (ii) the characteristic time of exciton recombination, $T_{1}$. The effects of the thermal bath will be considered in section IV.

One can verify that eq 6 can also be obtained by restriction to the $7 \times 7$ density matrix, from a Lindblad dynamics for the full $8 \times 8$ density matrix (eq 3 ). 
In the following, we fix $T_{1}=1 \mathrm{~ns}$, which is the exciton recombination time reported in the literature, ${ }^{24,25}$ and we focus on the effect of varying $T_{1 r}$.

\section{SUPERRADIANCE TRANSITION}

ST can be analyzed by studying the complex eigenvalues, $E_{r}=$ $E_{r}-i \Gamma_{r} / 2$ of $H_{\mathrm{e} f f}$, defined in eq 4 . As the coupling between the excitonic states and the $\mathrm{RC}$ increases, one observes a rearrangement of the widths, $\Gamma_{r}$ (the "superradiance" transition $\left.^{16}\right)$. We show this effect in Figure 1 (left panel),

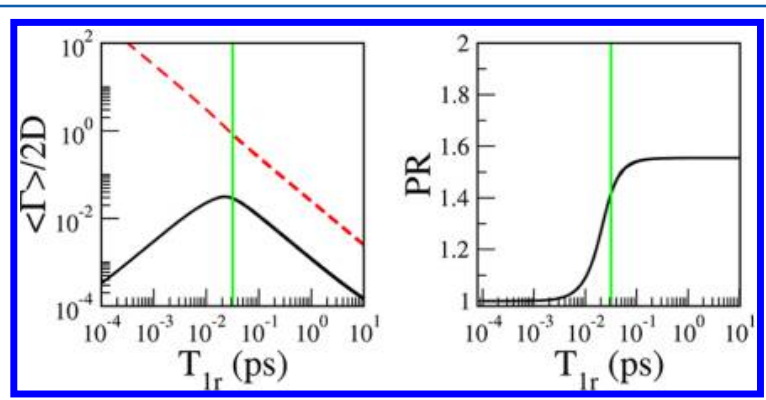

Figure 1. Left panel: average decay width, normalized to the mean level spacing, $D$, as a function of the coupling time to the RC, $T_{1 r}$. The black curve represents the average decay width of the 6 states with smallest width, while the dashed red curve shows the largest decay width. Right panel: PR of the eigenstate of $H_{\text {eff }}$ with the largest width as a function of $T_{1 \text { r. }}$ In both panels, the vertical (green) line indicates the critical value of $T_{1 r}$ at which ST occurs.

where the largest width (red dashed curve) and the average of the $7-1=6$ smallest widths (black full curve) are plotted as functions of $T_{1 r}$. For weak coupling to RC (large $T_{1 r}$ ), the widths of all states increase as $T_{1 r}$ decreases. On the other hand, below a critical value $T_{1 r}^{\mathrm{cr}}$, corresponding to ST (vertical line), the average of the 6 smallest widths decreases, while the largest width, corresponding to the superradiant state, increases. To examine localization of the excitation, we use the participation ratio $(\mathrm{PR})^{26}$ of a state $|\psi\rangle$, defined as

$$
\mathrm{PR}=\left(\sum_{i}|\langle i \mid \psi\rangle|^{4}\right)^{-1}
$$

Its value varies from 1 for fully localized to 7 for fully delocalized states. The right panel of Figure 1 shows the PR for the state associated with the largest width (the one decaying most quickly). In the superradiant regime, $T_{1 r}<T_{1 r}^{\mathrm{cr}}$, this state is fully localized on site 3 , the only site connected to the RC. For weak coupling to the RC, $T_{1 r}<T_{1 r}^{\mathrm{cr}}$, the PR is approximately 1.6. This small value, as compared to the maximal possible value of 7 , is explained by (Anderson) localization ${ }^{27}$ of the eigenstates on sites. The Anderson localization effect in the FMO system is due to the fact that the excitation energies of the sites and the couplings among them are all different. Thus, the FMO complex can be thought of as a disordered system. The critical value, $T_{1 r}^{\mathrm{cr}}$, at which ST occurs, can be estimated analytically. If all states have roughly the same width, at least for small coupling, then the superradiance condition coincides with that of overlapping resonances. Such reasoning can be applied to the FMO system, too. Here, eigenstates are mostly localized on the sites, and only site 3 is coupled to the RC. The widths are thus not uniform and most of the total width belongs to the eigenstate localized at site 3 . Imposing that the half-width, $\Gamma_{3} / 2$, is approximately equal to the mean level spacing $D, \Gamma_{3} / 2 \approx D$, and using $\Gamma_{3} \approx \hbar / T_{1 r}$, we obtain the critical value at which ST occurs

$$
T_{1 r}^{\mathrm{cr}} \approx \frac{\hbar}{2 D}
$$

In the FMO system, the energy level spacing is $D / h c \approx 83.5$ $\mathrm{cm}^{-1}$, which gives $T_{1 r}^{\mathrm{cr}} \approx 0.03 \mathrm{ps}$, a value in very good agreement with the numerical results of Figure 1 (vertical line).

Such a value, $T_{1 r}=0.03 \mathrm{ps}$, corresponds to a transfer rate, $\kappa$, from site 3 to the RC of $\kappa=1 /\left(2 T_{1 r}\right)=16.6 \mathrm{ps}^{-1}$. This value is larger than the values usually mentioned in the literature, which range from $0.25 \mathrm{ps}^{-1}$ to $4 \mathrm{ps}^{-1}$, 25 even if $1 \mathrm{ps}^{-1}$ is the most common value. ${ }^{5}$ This discrepancy can be due to the simplicity of our model, even if it is important to notice that, to the best of our knowledge, the real value of the coupling time, $T_{1 r}$, is not exactly known. In any case, for this reason, in section VIb, we consider a more realistic coupling scheme between the FMO system and the RC.

\section{EFFICIENCY OF ENERGY TRANSPORT IN THE PRESENCE OF A THERMAL BATH}

Interaction with the phonon environment is complicated, and it involves both dephasing and dissipation. ${ }^{3}$ Since superradiance is due to quantum coherence, in section IV A we first focus on dephasing and the consistent indirect relaxation, induced by the presence of classical noise. On the other hand, in section IV B, we consider both dephasing and dissipation induced by a finite temperature bath. Needless to say, while the latter bath induces at equilibrium a Gibbs energy level distribution, the former gives rise to an equal population of all energy levels.

A. Efficiency of Energy Transport in the Presence of Noise. As a first step, one can study the effects of the phonon bath modeling the thermal bath by a classical noise. In this case, the dephasing effects are adequately described using an interaction as in ref 24

$$
H_{\mathrm{SB}}=\sum q_{i}(t)|i\rangle\langle i|
$$

with

$$
\left\langle q_{i}(t) q_{j}(t)\right\rangle=\hbar^{2} \gamma_{\mathrm{d}} \delta_{i, j} \delta(t)
$$

where $\gamma_{\mathrm{d}}$ plays the role of the dephasing rate. This approach corresponds to an effective infinite temperature that leads to equal populations of energy levels at sufficiently large times. We take into account the interaction in eq 8 by adding a dephasing Lindblad operator to the master eq 6, as was done in ref 19. The interaction with noise leads to the Haken-Strobl master equation for the density matrix of the following form:

$$
\frac{\mathrm{d} \rho_{i, j}}{\mathrm{~d} t}=-\frac{i}{\hbar}\left(H_{\mathrm{eff}} \rho-\rho H_{\mathrm{eff}}^{+}\right)_{i, j}-\gamma_{\mathrm{d}}\left(1-\delta_{i, j}\right) \rho_{i, j}
$$

The first term in the r.h.s. of eq 10 takes into account the coherent evolution and the dissipation by recombination and trapping into the reaction center, (it is simply eq 6 rewritten in

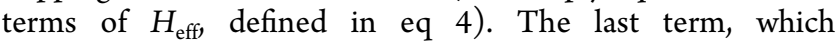
corresponds to the decay of the off-diagonal matrix elements, takes into account dephasing and indirect relaxation. For the FMO system, the amplitude of noise, $\hbar \gamma_{\mathrm{d}}$, is related to the temperature, $T$, by the relation, found in experiments ${ }^{2}$

$$
\hbar \gamma_{\mathrm{d}}(T) \simeq 0.52 \hbar c(T / K)(\mathrm{cm})^{-1}
$$

where $T$ is the temperature expressed in kelvin degrees, $K$. 
Transport efficiency has been measured as in ref 25 by the probability that the excitation is in the $\mathrm{RC}$ at the time $t_{\max }$

$$
\eta\left(t_{\max }\right)=\frac{1}{T_{1 r}} \int_{0}^{t_{\max }} \mathrm{d} t \rho_{33}(t)
$$

and by the average transfer time to the $\mathrm{RC}^{24}$

$$
\tau=\frac{1}{T_{1 r}} \int_{0}^{\infty} \mathrm{d} t t \rho_{33}(t) / \eta(\infty)
$$

In our simulations, we take the initial state

$$
\rho(0)=\frac{1}{2}(|1\rangle\langle 1|+| 6\rangle\langle 6|)
$$

since sites 1 and 6 receive the excitation from the antenna system. $^{24}$

It was numerically found in ref 20 that the efficiency reaches a maximum as a function of $T_{1}$. Here, we explain this as a consequence of ST, a general phenomenon in coherent quantum transport. In Figure 2, we plot $\eta\left(t_{\max }=5 \mathrm{ps}\right)$

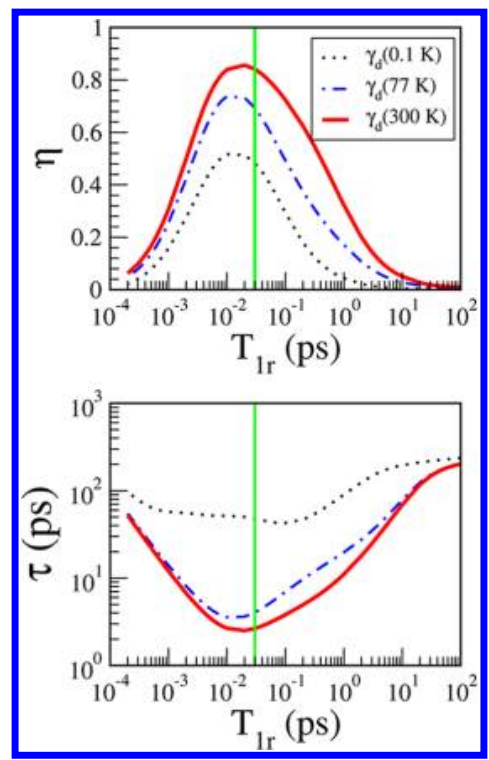

Figure 2. Upper panel: efficiency computed at $t_{\max }=5$ ps (see eq 12), as a function of $T_{1 r}$, for different dephasing rates (see eq 11). Lower panel: average transfer time (see eq 13) as a function of $T_{1}$, for the same effective temperatures. ST has been indicated as a green vertical line. The initial condition is $\rho(0)=(1 / 2)(|1\rangle\langle 1|+| 6\rangle\langle 6|)$.

(upper panel), and $\tau$ (lower panel), as functions of $T_{1}$. The maximum efficiency of energy transport (maximum $\eta$ and minimum $\tau$ ) is reached near the ST (vertical line). Note that $\eta\left(t_{\max }=5 \mathrm{ps}\right)$ has a maximum not only in the quantum limit $\left(\gamma_{\mathrm{d}}(T=0.1 \mathrm{~K})\right.$, black dashed curve $)$, but also considering the dephasing rate at room temperature $\left(\gamma_{\mathrm{d}}(T=300 \mathrm{~K})\right.$, red thick curve). These results show that the effects of the ST persist even in the presence of dephasing and indirect relaxation. Within the framework of the ST, the decrease in efficiency for large coupling to the $\mathrm{RC}$ can be interpreted as a localization effect (see Figure 1, right panel). Our results also show that dephasing can increase efficiency, since it counteracts quantum localization. This effect is known as environment-assisted quantum transport (ENAQT). ${ }^{24,25}$ The average transfer time (see Figure 2, lower panel) has a minimum near the ST on the order of a few picoseconds. This time is comparable with the transfer times estimated in the literature.
The coupling to the RC also induces a shift of the energy of site 3 (not only a decay width). ${ }^{16}$ This shift is assumed to be generically of the form $\delta=\varepsilon / T_{1 r}$, where $\varepsilon$ depends on the details of the coupling. We checked that the effect of changing $\varepsilon$ randomly, so as to produce up to a $50 \%$ change in the average level spacing, merely changes the efficiency at most by a few percent.

B. Efficiency of Energy Transport in the Presence of a Finite Temperature Thermal Bath. In this subection, we consider the effects of energy transport to the RC taking into consideration the interaction with a phonon bath at finite temperature, $T$, as described in ref 5 . Here, we consider that only site 3 is coupled to the RC, as described above. The Lindblad-type master equation has the form

$$
\frac{\mathrm{d} \rho_{i, j}}{\mathrm{~d} t}=-\frac{i}{\hbar}\left(H_{\mathrm{eff}} \rho-\rho H_{\mathrm{eff}}^{+}\right)_{i, j}+L_{p}(\rho)_{i j}
$$

where the action of the Lindblad operator on $\rho, L_{p}(\rho)$, is described in eq 5 of ref 5 . With this choice, at sufficiently large time, the transition to the Gibbs distribution occurs, in the absence of any other dissipative mechanism, such as the presence of "sinks".

In Figure 3 (upper panel), we present our results on the dependence of efficiency, $\eta$, as a function of $T_{1 r}$, for three bath

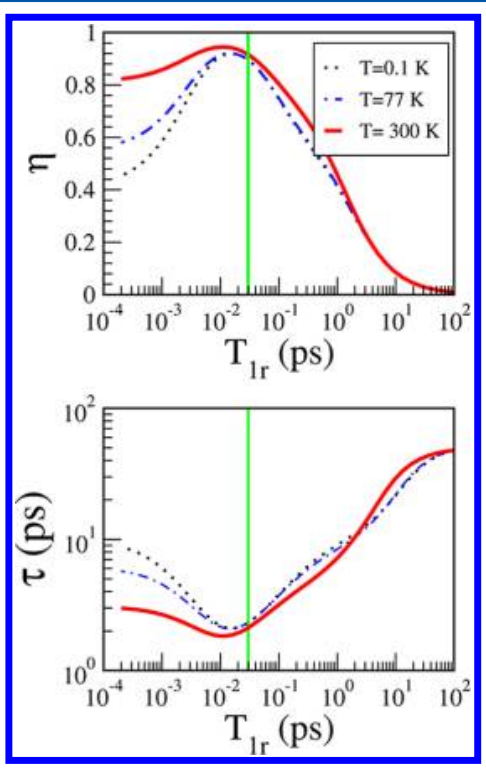

Figure 3. Upper panel: efficiency computed at $t_{\max }=5$ ps (see eq 12) as a function of $T_{1 r}$, for different temperatures of the phonon bath. Lower panel: average transfer time (see eq 13) as a function of $T_{1 r}$, for the same temperatures. ST has been indicated as a green vertical line. The initial condition is $\rho(0)=(1 / 2)(|1\rangle\langle 1|+| 6\rangle\langle 6|)$. We use as cutoff frequency of the spectral density of the thermal bath $\omega_{c}=150(\mathrm{~cm})^{-1}$, and a reorganization energy $E_{\mathrm{R}}=35(\mathrm{~cm})^{-1}$, where the latter two quantities have been defined in ref 5 .

temperatures. As one can see, at the ST, indicated as a vertical green line, the efficiency is about 0.92 , and it weakly depends on temperature. We also mention that the maximal efficiency occurs near the ST.

The value of $T_{1 r}$ at which one gets the maximal efficiency should not be confused with the average transfer time (see eq 13). In particular, in Figure 3 (lower panel), for parameters corresponding to the $S T$ and room temperature, $T_{1 r} \approx 30 \mathrm{fs}$ and $\tau \approx 2.1$ ps. 
Comparing Figure 2 and 3 (upper panels), one can see that the presence of the phonon bath significantly increases the efficiency of energy transport to the RC, almost without changing the position of its maximum. We also would like to mention that, in the presence of the thermal bath, the temperature effects on the efficiency are less significant than in the presence of a classical noise, as in Figure 2. Indeed, dissipation helps the system to reach the site 3 which has the lowest energy.

The analysis of this section shows that the consequences of the ST are very important even in the presence of dephasing and dissipation. For both models of thermal bath considered, the ST provides the maximal efficiency of energy transport. In the following, we will consider only the model of the phonon bath presented in section VI.A, which, as shown in this section, is sufficient to capture the main effects due to the phonon bath.

\section{QUANTUM VS CLASSICAL}

ST implies the presence of a maximum of the energy transport efficiency as a function of the coupling time to the RC, $T_{1 r}$. This effect is counterintuitive from a classical point of view. Indeed, the probability to escape (decay to the $\mathrm{RC}$ ) for a classical particle does not decrease as the escape rate $\left(1 / T_{1 r}\right.$ from site 3$)$ is increased. In order to demonstrate the difference between the effects of quantum coherence on energy transfer discussed above, and the corresponding classical energy transport, we consider a classical master equation for the population dynamics, as in the Forster approach ${ }^{28}$

$$
\frac{\mathrm{d} P_{i}}{\mathrm{~d} t}=\sum_{k}\left(T_{i, k} P_{k}-T_{k, i} P_{i}\right)-\frac{P_{i}}{T_{1}}-\delta_{i, 3} \frac{P_{i}}{T_{1 r}}
$$

where $P_{i}$ is the probability to be on site $i, T_{i, k}$ is the transition matrix, and the last two terms take into account the possibility for the classical excitation to escape the system. The transition rates from site $i$ to site $k$ have been computed from ref 29, neglecting the dependence on the coupling to the RC (for a classical particle, the probability to go from any site to site 3 does not depend on the coupling to the RC).

The comparison between classical and quantum behavior is shown in Figure 4 (upper panel). The classical dynamics leads to a very different dependence of the efficiency on $T_{1 r}$. Namely, the efficiency in the classical case does not exhibit a maximum but simply decays with $T_{1}$. This shows that the ST effect is due to quantum coherence only.

\section{DIFFERENT COUPLING SCHEMES}

So far, we have considered site 3 to be the only one coupled to the RC. However, it is not known for sure which sites are connected to the RC, even though sites 3 and 4 are the most likely candidates, since they are closest to the RC. ${ }^{24}$ As mentioned above, the non-Hermitian Hamiltonian formalism easily allows one to describe different coupling schemes, which can be included in the effective Hamiltonian (eq 4) by properly choosing the coupling transition amplitude, $A_{i}^{\mathrm{RC}}$, between the sites of the FMO complex and the RC. Note that, while in the previous section we indicated the channel in the $\mathrm{RC}$ with the number 8 , here we label it as RC.

A. Coupling from Sites 3 and 4. Since, to the best of our knowledge, it is not exactly known from experimental data how the energy transport occurs, in the following we choose three different situations, showing that the essential features of the phenomenon indicated in the previous section do not change

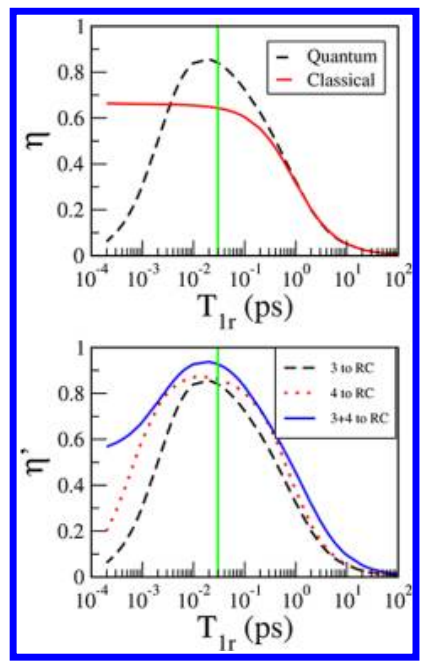

Figure 4. Upper panel: quantum and classical efficiency computed at $t_{\max }=5 \mathrm{ps}$, as a function of $T_{1 r}$ at room temperature dephasing rate, $\gamma_{\mathrm{d}}(T=300 \mathrm{~K})$ (see eq 11$)$. Lower panel: efficiency computed at $t_{\max }=$ 5 ps, using eq 16 , as a function of $T_{1 r}$, at room temperature dephasing rate, $\gamma_{\mathrm{d}}(T=300 \mathrm{~K})$, for different coupling to $\mathrm{RC}$. The vertical green line represents ST. As initial conditions, we choose $\rho(0)=(1 / 2)($ I $1\rangle\langle 1|+| 6\rangle\langle 6|)$.

too much. Specifically, we consider the following: only site 3 is coupled to RC, so that we set $A_{3}^{\mathrm{RC}}=\left[\hbar /\left(2 T_{1 r}\right)\right]^{1 / 2}$ (as done above); only site 4 is coupled to the RC, so we set $A_{4}^{\mathrm{RC}}=[\hbar /$ $\left.\left(2 T_{1 r}\right)\right]^{1 / 2}$; both sites 3 and 4 are coupled to the RC, so we set $A_{3}^{\mathrm{RC}}=A_{4}^{\mathrm{RC}}=\left[\hbar /\left(2 T_{1 r}\right)\right]^{1 / 2}$.

In a general setting, the probability for the excitation to be in the $\mathrm{RC}$ at time $t_{\max }=5$ ps cannot be computed using eq 12 , since by merely summing that expression for each site connected to the RC, we neglect interference effects. The efficiency should be computed using

$$
\eta^{\prime}\left(t_{\max }\right)=1-\operatorname{Tr}\left(\rho\left(t_{\max }\right)\right)-\frac{1}{T_{1}} \int_{0}^{t_{\max }} \mathrm{d} t \operatorname{Tr}(\rho(t))
$$

Here, $1-\operatorname{Tr}\left(\rho\left(t_{\max }\right)\right)$ is the probability that the excitation leaves the system by the time $t_{\max }$. The last term in eq 16 is the probability that the excitation has been lost by recombination during this time. If there is just one site coupled to the RC, then eq 16 reduces to eq 12 .

In Figure 4 (lower panel), we show that the efficiency is sensitive to different coupling schemes. In particular, we notice that coupling through site 4 achieves a greater efficiency than coupling through site 3 . If both sites are coupled to the RC, then the efficiency is further improved, and the decay for small coupling times is smaller than that for a single coupled site.

B. Efficiency vs Position of the RC. In the following, we consider a more realistic coupling scheme to RC, namely, the case in which all sites of the FMO system have an electric dipole coupling to the same channel in the RC. This assumption can be justified, since in the $\mathrm{RC}$, there are the same bacteriochlorophyll (BChl) molecules which compose the FMO system. So, it is reasonable to assume that the excitation is transferred to the $\mathrm{RC}$ by the same mechanism that operates between the $\mathrm{BChl}$ molecules in the FMO system.

The electric dipole transition amplitude from site $i$ to the RC can be written as

$$
V_{i}^{\mathrm{RC}}=\frac{C}{R_{i, \mathrm{RC}}^{3}}\left[\vec{\mu}_{i} \cdot \vec{\mu}_{R C}-3\left(\vec{\mu}_{i} \cdot \hat{R}_{i, \mathrm{RC}}\right)\left(\vec{\mu}_{\mathrm{RC}} \cdot \hat{R}_{i, \mathrm{RC}}\right)\right]
$$


where $R_{i, \mathrm{RC}}$ is the distance from site $i$ to the $\mathrm{RC}, \mu_{i}$ is the dipole moment at the site $i$, and $\mu_{\mathrm{RC}}$ is the dipole moment assigned to the RC. We take the position of the BChls and their dipole moments from ref 20. Here, we assume that the coupling strength between the sites and the RC is equal to that between the sites, so that $C|\mu|^{2}=134000 \mathrm{~cm}^{-1}(\AA)^{3}$, as in ref 20 .

In order to determine the coupling amplitude from site $i$ to the continuum of states in the RC, we evaluate the transfer rate, $\kappa_{i}$ from the Fermi-golden rule: ${ }^{5}$

$$
\kappa_{i}=\frac{2 \pi \rho_{\mathrm{RC}}\left|V_{i}^{\mathrm{RC}}\right|^{2}}{\hbar}
$$

where $\rho_{R C}$ represents the density of states in the RC. It is interesting to observe that an expression for the transfer rate similar to eq 18 can also be obtained without perturbation theory (see for instance ref 30, where the continuum was modeled as a semi-infinite lead). We can now determine the non-Hermitian Hamiltonian (eq 4), setting

$$
A_{i}^{\mathrm{RC}}=\sqrt{2 \pi \rho_{\mathrm{RC}}} V_{i}^{\mathrm{RC}}
$$

Note that now the coupling between the FMO complex and the RC depends on the position of the RC. In order to determine how the efficiency of energy transfer depends on the position of the RC with respect to the FMO complex, we assume $\vec{\mu}_{\mathrm{RC}}=\vec{\mu}_{3}$ and place the RC in the same $y$ and $z$ positions of site 3 , as given in ref 20 . The transport efficiency has been studied by varying the distance, $d$, from site 3 along the $x$ direction. In order to compute the transition amplitude, $A_{i}^{\mathrm{RC}}$, we need the density of states of the RC which, to the best of our knowledge, is not known experimentally. For this reason, we consider different densities of states, respectively, larger, equal, or smaller than the density of states of the FMO system, $\rho_{\text {FMO }} \simeq 1 / D$, with $D$ being the mean level spacing of the FMO complex discussed in section III.We show in Figure 5 how the

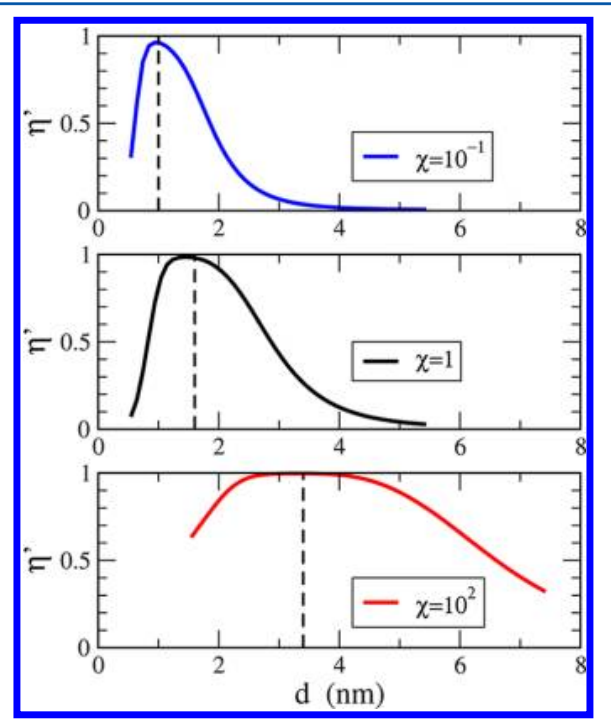

Figure 5. Efficiency (eq 16) computed at $t_{\max }=5$ ps vs the distance $d$ (nm) of the RC from site 3 in the FMO complex for different ratios $\chi$ $=\rho_{\mathrm{RC}} / \rho_{\mathrm{FMO}}$. Here, an electric dipole coupling between the RC and the FMO system (see eq 17) has been considered. The vertical dashed lines, obtained from eq 19, represent the critical distances at which superradiance transition occurs. As initial conditions, we choose $\rho(0)$ $=(1 / 2)(|1\rangle\langle 1|+| 6\rangle\langle 6|)$. Data in this figure refer to a roomtemperature dephasing rate, $\gamma_{\mathrm{d}}(T=300 \mathrm{~K}$ ) (see eq 11). efficiency, computed with eq 16 using eq 10 , varies as a function of the distance from the RC to site 3 of the FMO system. In Figure 5, we consider a room-temperature dephasing rate $\gamma_{\mathrm{d}}(T=300 \mathrm{~K})$, for different ratios, $\chi=\rho_{\mathrm{RC}} / \rho_{\mathrm{FMO}}=0.1,1$, $10^{2}$, respectively, from the upper to the lower panel. As one can see, the optimal distance, which is the distance that maximizes the efficiency, slowly depends on the density of states in the RC.

We can use the superradiant criterium obtained in eq 7 to get an analytical expression for the optimal distance of the $\mathrm{RC}$ from the FMO complex. Since site 3 is the closest to the RC, we can use eq 7 , and we find that the ST occurs for $\hbar \kappa_{3}=D$. Finally, from eq 18 we have

$$
d_{\max }=\left(2 \pi B^{2}\right)^{1 / 6}\left(\rho_{\mathrm{RC}} \rho_{\mathrm{FMO}}\right)^{1 / 6}
$$

where

$$
B=C\left[\vec{\mu}_{3} \cdot \vec{\mu}_{R C}-3\left(\vec{\mu}_{3} \cdot \hat{R}_{3, \mathrm{RC}}\right)\left(\vec{\mu}_{\mathrm{RC}} \cdot \hat{R}_{3, \mathrm{RC}}\right)\right]
$$

Equation 19 gives us the distance at which the superradiance transition occurs. The critical distance obtained from eq 19 is shown in Figure 5 as dashed vertical lines. As one can see, the estimate is very good. Note that changing the density of states in the RC from $\rho_{\mathrm{FMO}} / 10$ to $10^{2} \rho_{\mathrm{FMO}}$ only changes the optimal distance from $1 \mathrm{~nm}$ to $3 \mathrm{~nm}$. These distances are consistent with available structural data for the RC-FMO complex (see for instance ref 31). The result is remarkable, since it shows that the superradiant criterium suffices to determine the optimal distance from the RC to the FMO complex for a wide range of values for the density of states of the RC.

\section{CONCLUSION}

We have analyzed energy transport in the FMO system with the aid of a non-Hermitian Hamiltonian approach. This allows us to take into account the effect of the coupling of the FMO system to the reaction center in a consistent way, not merely phenomenologically, as is usually done in the literature. We have shown that, by increasing the strength of the coupling to the reaction center, a superradiance transition occurs. This transition occurs at approximately the same value of the coupling for which energy transport efficiency is maximal. Indeed, the superradiance transition is due to coherent constructive interference between the paths to the RC, and this effect enhances the rate of energy transfer. Since the ST effect is due to quantum coherence, one might expect that any consequences of ST would disappear in the presence of dephasing and relaxation provided by the thermal bath. On the contrary, we have shown that the effect of superradiance survives in the presence of the thermal bath, and the maximal efficiency only depends weakly on temperature. We have also estimated the ST critical value analytically. For coupling strengths of the FMO system to the RC near the critical one, where the superradiance transition takes place, we obtained average energy transfer times comparable to experimental values (a few picoseconds). Finally, we took into account a realistic coupling scheme between the FMO system and the $\mathrm{RC}$, and derived from the superradiance condition the analytical expression, eq 19, for the optimal distance from the RC to the FMO complex. This analytical expression depends on the density of state in the RC. Within a wide range of densities of state, the optimal distance which we obtained analytically is approximately a few nanometers and is consistent 
with available structural data on RC. Note also that eq 19 is valid for a generic Donor-Acceptor complex.

Our analysis shows that the superradiance mechanism might play an important role in explaining the efficiency of quantum transport in photosynthetic light-harvesting systems and in engineering artificial light-harvesting systems.

\section{AUTHOR INFORMATION}

\section{Corresponding Author}

*E-mail nicedirac@gmail.com.

\section{Notes}

The authors declare no competing financial interest.

\section{ACKNOWLEDGMENTS}

This work has been supported by Regione Lombardia and CILEA Consortium through a LISA (Laboratory for Interdisciplinary Advanced Simulation) Initiative (2010/11) grant [http://lisa.cilea.it]. Support from grant D.2.2 (2010) from Università Cattolica is also acknowledged. The work by G.P.B. was carried out under the auspices of the National Nuclear Security Administration of the U.S. Department of Energy at the Los Alamos National Laboratory under Contract No. DEAC52- 06NA25396. M.M. has been supported by the NSERC Discovery Grant No. 205247. F.B., M.M., and G.P.B thank the Institut Henri Poincare (IHP) for partial support at the final stage of this work.

\section{REFERENCES}

(1) Engel, G. S.; Calhoun, T. R.; Read, E. L.; Ahn, T.-K.; Mancal, T.; Cheng, Y.-C.; Blankenship, R. E.; Fleming, G. R. Nature 2007, 446, 782786.

(2) Panitchayangkoona, G.; Hayesa, D.; Fransteda, K. A.; Carama, J. R.; Harela, E.; Wenb, J.; Blankenshipb, R. E.; Engela, G. S. Proc. Natl. Acad. Sci. U.S.A. 2010, 107, 12766-12770.

(3) Sarovar, M.; Ishizaki, A.; Fleming, G. R.; Whaley, K. B. Nat. Phys. 2010, 6, 462-7.

(4) Hossein-Nejad, H.; Scholes, G. D. New J. Phys. 2010, 12, 065045.

(5) Mohseni, M.; Rebentrost, P.; Lloyd, S.; Aspuru-Guzik, A. J. Chem. Phys. 2008, 129, 174106-9.

(6) Lloyd, S.; Mohseni, M. New J. Phys. 2010, 12, 075020.

(7) Scholes, G. D. Chem. Phys. 2002, 275, 373-386.

(8) Dicke, R. H. Phys. Rev. 1954, 93, 99-110.

(9) Sokolov, V. V.; Zelevinsky, V. G. Nucl. Phys. 1989, A504, 562588; Phys. Lett. B 1988, 202, 10-14. Rotter, I. Rep. Prog. Phys. 1991, 54, 635 .

(10) Sokolov, V. V.; Zelevinsky, V. G. Ann. Phys. (N.Y.) 1992, 216, 323-350.

(11) Pudlak, M.; Pichugin, K. N.; Nazmitdinov, R. G.; Pincak, R. Phys. Rev. E 2011, 84, 051912-6.

(12) Verbaarschot, J. J. M.; Weidenmüller, H. A.; Zirnbauer, M. R Phys. Rep. 1985, 129, 367-438. Lehmann, N.; Saher, D.; Sokolov, V. V.; Sommers, H.-J. Nucl. Phys. 1995, A582, 223-256. Fyodorov, Y. V.; Sommers, H.-J. J. Math. Phys. 1997, 38, 1918-1982. Sommers, H.-J.; Fyodorov, Y. V.; Titov, M. J. Phys. A: Math. Gen. 1999, 32, L77.

(13) Celardo, G. L.; Izrailev, F. M.; Zelevinsky, V. G.; Berman, G. P. Phys. Lett. B 2008, 659, 170-175. Celardo, G. L.; Izrailev, F. M.; Zelevinsky, V. G.; Berman, G. P. Phys. Rev. E 2007, 76, 031119-12. Celardo, G. L.; Sorathia, S.; Izrailev, F. M.; Zelevinsky, V. G.; Berman, G. P. CP995, Nuclei and Mesoscopic Physics, Danielewicz, P., Piecuch, P., Zelevinsky, V. G.,Eds.; WNMP, 2007.

(14) Volya, A.; Zelevinsky, V. G. Phys. Rev. C 2003, 67, 054322-11; Phys. Rev. Lett. 2005, 94, 052501-4; Phys. Rev. C 2006, 74, 06431417.

(15) Stöckmann, H.-J.; Persson, E.; Kim, Y.-H.; Barth, M.; Kuhl, U.; Rotter, I. Phys. Rev. E 2002, 65, 066211-10. Nazmitdinov, R. G.; Sim, H.-S.; Schomerus, H.; Rotter, I. Phys. Rev. B 2002, 66, 241302-4.
(16) Celardo, G. L.; Kaplan, L. Phys. Rev. B 2009, 79, 155108-9. Celardo, G. L.; Smith, A. M.; Sorathia, S.; Zelevinsky, V. G.; Senâ€kov, R. A.; Kaplan, L. Phys. Rev. B 2010, 82, 165437-12.

(17) Sadreev, A. F.; Rotter, I. J. Phys. A 2003, 36, 11413.

(18) Renaud, N.; Ratner, M. A.; Mujica, V. J. Chem. Phys. 2011, 135, 075102-9.

(19) Mahaux, C.; Weidenmüller, H. A. Shell Model Approach to Nuclear Reactions; North Holland: Amsterdam, 1969.

(20) Mohseni, M.; Shabani, A.; Lloyd, S.; Rabitz, H.; Shabani, A., Mohseni, M., Rabitz, H., Lloyd, S. Phys. Rev. E, 2012, 86, 011915-15; arXiv: $1104.4812 \mathrm{v} 1$ [quant-ph].

(21) Recent studies reported the presence of an additional Bchl pigment per subunit: Ben-Shem, A.; Frolow, F.; Nelson, N. FEBS Lett. 2004, 564, 274-280. Tronrud, D. E.; Wen, J. Z.; Gay, L.; Blankenship, R. E. Photosynth. Res. 2009, 100, 79-87 These new findings do not change the main conclusions of our analysis.

(22) Milonni, P. W.; Knight, P. L. Phys. Rev. A 2009, 10, 1096-1108.

(23) Messiah, A. Quantum Mechanics, English ed.; North Holland Publishing Company: Amsterdam, 1962; Vol II, Chapter XXI.13.

(24) Rebentrost, P.; Mohseni, M.; Kassal, I.; Lloyd, S.; Aspuru-Guzik, A. New J. Phys. 2009, 11, 033003 . Rebentrost, P.; Mohseni, M.; Aspuru-Guzik, A. J. Phys. Chem. B 2009, 113, 9942-9947.

(25) Plenio, M. B.; Huelga, S. F. New J. Phys. 2008, 10, 113019. Caruso, F.; Chin, A. W.; Datta, A.; Huelga, S. F.; Plenio, M. B. J. Chem. Phys. 2009, 131, 105106-15.

(26) Izrailev, F. M.; Krokhin, A. A.; Makarov, N. M. Phys. Rep. 2012, $512,125-254$.

(27) Anderson, P. W. Phys. Rev. 1958, 109, 1492-1505.

(28) Forster, Th. Modern Quantum Chemistry, Sinannouglu, O., Ed.; Academic Press: New York, 1965; p 93.

(29) Leegwater, J. A. J. Phys. Chem. 1996, 100, 14403-14409.

(30) Sadreev, A. F.; Rotter, I. J. Phys. A 2003, 36, 11413.

(31) Remigy, H.-W.; Stahlberg, H.; Fotiadis, D.; MÃ1/4ller, S. A.; Wolpensinger, B.; Engel, A.; Hauska, G.; Tsiotis, G. J. Mol. Biol. 1999, 290, 851-858. Remigy, H.-W.; Hauska, G.; Muller, S. A.; Tsiotis, G. Photosynthesis Res. 2002, 71, 91-98. 